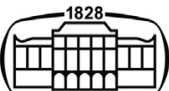

AKADÉMIAI KIADÓ

IMAGING

\section{CASE REPORT}

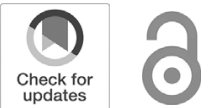

\title{
Thrombosed orbital varix with mild, spontaneously resolving symptoms
}

\section{MÁTÉ MAGYAR ${ }^{1,3}$, PÉTER BARSI ${ }^{1}$, KATALIN KORÁNYI ${ }^{4}$ and PÁL NOVÁK KAPOSI ${ }^{2 *}$}

\author{
${ }^{1}$ Department of Neuroradiology, Faculty of Medicine, Medical Imaging Centre, Semmelweis \\ University, Budapest, Hungary \\ ${ }^{2}$ Department of Radiology, Medical Imaging Centre, Faculty of Medicine, Semmelweis University, \\ Budapest, Hungary \\ ${ }^{3}$ Department of Neurology, Faculty of Medicine, Semmelweis University, Budapest, Hungary \\ ${ }^{4}$ Department of Ophthalmology, Faculty of Medicine, Semmelweis University, Budapest, Hungary
}

Received: May 19, 2020 • Accepted: May 25, 2020

\section{ABSTRACT}

Orbital varices are abnormally enlarged, thin walled veins in direct connection with normal orbital vessels. Stooping, bending, coughing or straining increase the venous tension and in this way can cause symptoms, such as bulging of the eyes (proptosis), orbital pain, bleeding, intermittent double vision. Small varices are managed by observation while larger lesions may require surgery. In our case report we demonstrate the case of a 72-year-old female patient with right sided retro-orbital pressure sensation for weeks, and exophthalmos with blurred vision for 1 week. MR investigation proved the presence of a partially thrombosed orbital varix on the right side, and a smaller, non-thrombosed varix on the left side. Observation was agreed, and the symptoms resolved spontaneously after several weeks.

\section{KEYWORDS}

Orbital varix, bilateral orbital varices, proptosis, exophthalmos, visual disturbance

\section{Introduction}

Orbital varix is a rare entity that occurs equally in males and females, typically in the 2nd or 3 rd decade of life [1]. It results from the congenital weakness of the postcapillary venous wall [2]. The weakness leads to the proliferation and dramatic dilation of these valveless, thin walled orbital veins. Presenting symptoms of the venous tension and the conflict of vascular formations with oculomotor muscles can include intermittent diplopia, proptosis, decreased visual acuity, and retro-orbital pain [2-4]. A lot of conditions, which increase the venous tension (forward bending, coughing, breath holding, Valsalva maneuver) can cause dramatic protrusion of the affected eye, called unilateral stress proptosis [5]. In some cases orbital varices can manifest with thrombosis and hemorrhage, producing a more sustained, severe proptosis and in rare cases optic nerve injury with consequential visual impairment [6].

*Corresponding author. Department of Radiology, Medical Imaging Centre, Semmelweis University, Korányi Sándor u. 2., 1083 Budapest, Hungary. Tel.: +36 (20) 6632151 kaposi.pal@mail.semmelweis-univ.hu

\section{Case report}

A 72-year-old female patient was referred to the Department of Ophthalmology, with several weeks' history of right retro-orbital pressure during forward bending. She did not have any previous illnesses, only presbyopia. Physical examination was normal, there was no 


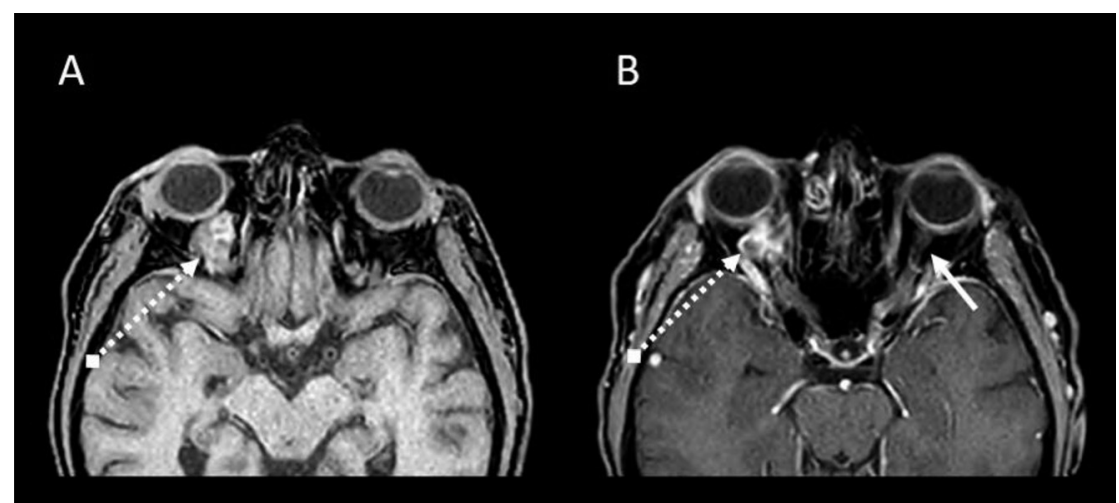

A

B
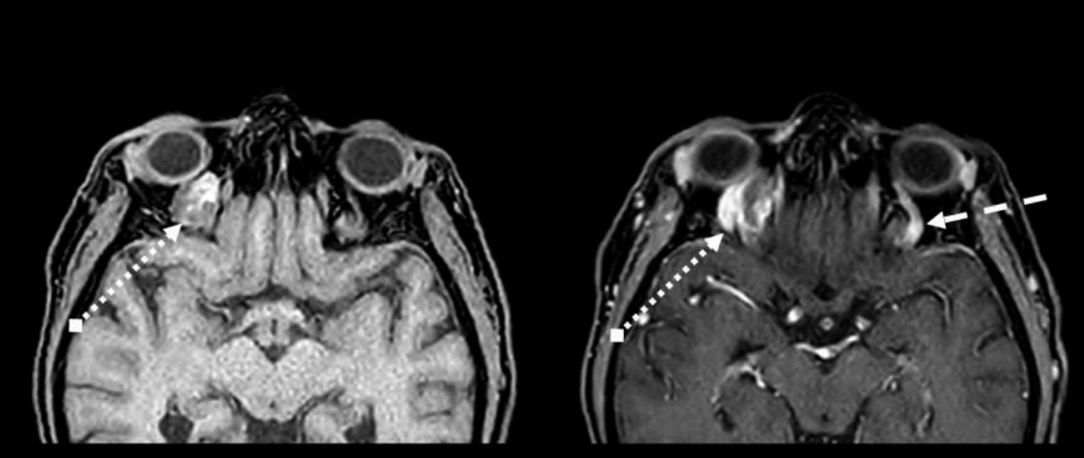

Figure 1. T1-weighted High Resolution Isotropic Volume Examination (THRIVE) without contrast (A) and with contrast (B). Arrow with continuous line: intact optic nerve; Arrow with dotted line: superior ophthalmic vein on the left side; Arrow with dotted stem: orbital varix

exophthalmos, her best corrected visual acuity was full on both sides, visual fields were intact, pupillary responses were equal and fundoscopy was unremarkable. Eye movements were in normal range, intraocular pressure was also normal according to Goldmann tonometry. The patient was released and further investigations were planned. After a week the patient felt swelling of the right upper eyelid, pain in the forehead, and blurring of the vision of the right eye. At the Department of Ophthalmology exophthalmos (3 mm), periorbital edema and hyperemia were found on the right side.

The ophthalmologist requested an orbital MR investigation with contrast material (Philips Ingenia $1.5 \mathrm{~T}$, contrast material: Gadovist $7 \mathrm{~mL}$ ), which revealed a rightsided intraconal thrombosed varix $(20 \times 17 \times 10 \mathrm{~mm})$ displacing the optic nerve, but the thickness and signal intensity of the nerve were normal (Figs. 1 and 2). The leftsided superior orbital vein was also mildly dilatated (3-4 $\mathrm{mm})$. There were no abnormal enhancing mass lesions within the orbits or brain parenchyma. No intracranial arteriovenous malformations, dural fistulas, or carotid cavernous fistulas were identified. Targeted orbital color Doppler investigation on the right side found a hypoechoic retrobulbar lesion without flow signal which mildly displaced the optic nerve.

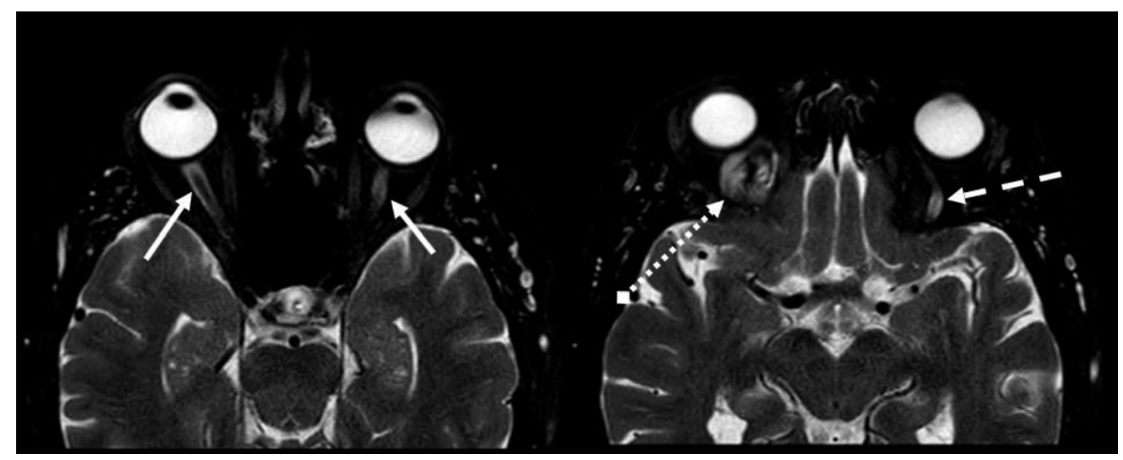

Figure 2. T2-weighted images without abnormal signal intensity of the optic nerve. Arrow with continuous line: optic nerve; Arrow with dotted line: superior ophthalmic vein on the left side; Arrow with dotted stem: varix 
Two weeks later the symptoms of the patient resolved spontaneously. The control ophtalmogical investigation was normal, no exophthalmos, periorbital edema, or hyperemia was found.

\section{Discussion}

The best diagnostic tool for orbital varices is magnetic resonance imaging. Orbital varices have hypo-to hyperintense signal on T1-weighted images, hyperintense signal on T2-weighted images, and usually enhance intensely after the administration of contrast material. The post-contrast sequences are also essential to prove thromboembolic complications [7]. Other causes or orbital masses, such as cavernous hemangiomas and lymphangioma should be included in the differential diagnosis of orbital varices [7].

In our case MRI demonstrated a tortuous, partially thrombosed - can explain why flow signal cannot be detected with color Doppler investigation - dilatated intraconal vessel in the posterior orbit, which tapered toward the orbital apex and lacked internal septae. Imaging did not reveal orbital wall defect.

Observation was agreed, and the symptoms of the patient resolved spontaneously.

There are no well established guidelines for treatment. Small lesions do not usually require treatment, but observation. Recurrent thrombosis, pain, severe proptosis, or compression of the optic nerve require intervention, for example electrothrombosis [8], stereotactic gamma knife radiosurgery [9], sclerotherapy, endovascular embolization with or without surgical excision. The long-term risk of missed treatment are optic nerve atrophy and blindness $[3,8,10]$.

Funding sources: No financial support was received for this case report.

Authors' contribution: All persons who meet authorship criteria are listed as authors, and all authors certify that they have participated sufficiently in the work to take public responsibility for the content, including participation in the concept, writing, or revision of the manuscript. Acquisition of images: PB, PKN, MM. Analysis and/or interpretation of literature: PB, PKN, MM. Drafting the manuscript: MM.
Revising the manuscript critically for important intellectual content: BP, PKN, KK. Approval of the version of the manuscript to be published: BP, PNK, KK, MM. This manuscript has not been published previously and is not under consideration for publication elsewhere.

Conflict of interests: The authors have no conflict of interest to disclose.

\section{REFERENCES}

[1] Rubin PAD, Remulla HD: Orbital venous anomalies demonstrated by spiral computed tomography. Ophthalmology 1997; 104(9): 1463-70.

[2] Smoker WR, Gentry LR, Yee NK, Reede DL, Nerad JA: Vascular lesions of the orbit: more than meets the eye. Radiographics 2008; 28(1): 185-204.

[3] Carrim ZI, Ahmed TY, Wykes WN: Isolated superior ophthalmic vein thrombosis with orbital congestion: a variant of idiopathic orbital inflammatory disease? Eye (Lond). England 2007; 21(5): 665-6.

[4] Vadlamudi V, Gemmete JJ, Chaudhary N, Pandey AS, Kahana A: Transvenous sclerotherapy of a large symptomatic orbital venous varix using a microcatheter balloon and bleomycin. BMJ Case Rep 2015.

[5] Cohen JA, Char DH, Norman D: Bilateral orbital varices associated with habitual bending. Arch Ophthalmol 1995; 113(11): 1360-2.

[6] Phan IT, Hoyt WF, McCulley TJ, Hwang TN: Blindness from orbital varices: case report. Orbit 2009; 28(5): 303-5.

[7] Heran F, Berges O, Blustajn J, Boucenna M, Charbonneau F, Koskas P: Tumor pathology of the orbit. Diagn Interv Imaging 2014; 95(10): 933-44.

[8] Tsai AS, Fong KS, Lim W, Al Jajeh I, Chuah CT, Rootman J: Bilateral orbital varices: an approach to management. Ophthalmic Plast Reconstr Surg 2008; 24(6): 486-8.

[9] Xu D, Liu D, Zhang Z, Zhang Y, Song G: Gamma knife radiosurgery for primary orbital varices: a preliminary report. Br J Ophthalmol 2011; 95(9): 1264-7.

[10] Kumar RR, Singh A, Abhishek: Embolization of a deep orbital varix through endovascular route. Indian J Ophthalmol 2015; 63(3): $270-2$. 